\title{
Review Article \\ The Multifaceted Functions of CXCL10 in Cardiovascular Disease
}

\author{
Pleunie van den Borne, ${ }^{1}$ Paul H. A. Quax, ${ }^{2,3}$ Imo E. Hoefer, ${ }^{1,4}$ and Gerard Pasterkamp ${ }^{1,4}$ \\ ${ }^{1}$ Laboratory of Experimental Cardiology, University Medical Center Utrecht, P.O. Box 85500, 3508 GA, Utrecht, The Netherlands \\ ${ }^{2}$ Department of Surgery, Leiden University Medical Center, P.O. Box 9600, 2300 RC, Leiden, The Netherlands \\ ${ }^{3}$ Einthoven Laboratory of Experimental Vascular Medicine, Leiden University Medical Center, P.O. Box 9600, 2300 RC, \\ Leiden, The Netherlands \\ ${ }^{4}$ Interuniversity Cardiology Institute of the Netherlands, P.O. Box 19258, 3501 DG, Utrecht, The Netherlands
}

Correspondence should be addressed to Gerard Pasterkamp; g.pasterkamp@umcutrecht.nl

Received 22 January 2014; Accepted 6 March 2014; Published 23 April 2014

Academic Editor: Koji Ikeda

Copyright (C) 2014 Pleunie van den Borne et al. This is an open access article distributed under the Creative Commons Attribution License, which permits unrestricted use, distribution, and reproduction in any medium, provided the original work is properly cited.

C-X-C motif ligand 10 (CXCL10), or interferon-inducible protein-10, is a small chemokine belonging to the CXC chemokine family. Its members are responsible for leukocyte trafficking and act on tissue cells, like endothelial and vascular smooth muscle cells. CXCL10 is secreted by leukocytes and tissue cells and functions as a chemoattractant, mainly for lymphocytes. After binding to its receptor CXCR3, CXCL10 evokes a range of inflammatory responses: key features in cardiovascular disease (CVD). The role of CXCL10 in CVD has been extensively described, for example for atherosclerosis, aneurysm formation, and myocardial infarction. However, there seems to be a discrepancy between experimental and clinical settings. This discrepancy occurs from differences in biological actions between species (e.g. mice and human), which is dependent on CXCL10 signaling via different CXCR3 isoforms or CXCR3-independent signaling. This makes translation from experimental to clinical settings challenging. Furthermore, the overall consensus on the actions of CXCL10 in specific CVD models is not yet reached. The purpose of this review is to describe the functions of CXCL10 in different CVDs in both experimental and clinical settings and to highlight and discuss the possible discrepancies and translational difficulties. Furthermore, CXCL10 as a possible biomarker in CVD will be discussed.

\section{Introduction}

Chemokines are soluble low molecular weight proteins that are involved in a wide variety of processes during physiological and pathological conditions. They can be secreted by and act on different cell types depending on the expression of specific receptors. Chemokines are known to be involved in leukocyte trafficking but can also act on other cells like endothelial cells and vascular smooth muscle cells (VSMCs) [1]. Subgroups of chemokines that have been identified are $\mathrm{C}, \mathrm{CC}, \mathrm{CX} 3 \mathrm{C}$, and $\mathrm{CXC}$, based on molecular structure and arrangement of cysteine residues that form disulfidebonding pairs. C chemokines mainly recruit lymphocytes, while CC chemokines recruit monocytes. So far, only one CX3C chemokine has been described. CX3CL1 (fractalkine) can act as a chemoattractant for different leukocytes (soluble CX3CL1) and promotes cell adhesion to activated endothelial cells (cell-bound CX3CL1). The last family of chemokines, the CXC chemokines, is involved in leukocyte trafficking and endothelial and vascular smooth muscle cell (VSMC) proliferation and motility [2-4]. In this review, the role of C-X-C motif ligand 10 (CXCL10) in different cardiovascular disease models will be highlighted. CXCL10 belongs to the CXC chemokine family [4]. The CXC chemokines can be subdivided into two groups according to the presence or absence of a tripeptide glutamic acid-leucine-arginine (GluLeu-Arg motif; "ELR") motif preceding the first conserved cysteine: the ELR motif positive (ELR+) and ELR motif negative (ELR-) CXC chemokines. ELR+ CXC chemokines are known to attract neutrophils and hold more angiogenic 
properties, whereas ELR- CXC chemokines are lymphocyte attractants with angiostatic properties [5, 6]. CXCL10 belongs to the ELR- CXC chemokines and is also known as interferon-inducible protein-10 (IP-10). As the name implies, this chemokine can be secreted upon interferon gamma (IFN $\gamma$ ) production by a wide variety of cell types, such as endothelial cells, fibroblasts, keratinocytes, monocytes, and T lymphocytes [7], but secretion can also be induced by lipopolysaccharide and proinflammatory cytokines such as IFN-alpha and IFN-beta as well as tumor necrosis factoralpha $[8,9]$, depending on the cell type. CXCL10 exerts its biological effects mainly via binding to CXCR3 [10]. Also, CXCR3-independent pathways have been studied for CXCL10 involving binding of CXCL10 to heparan sulfate glycosaminoglycans (GAGs) [11-14] for cells not expressing CXCR3. CXCL10 not only induces chemoattraction of inflammatory cells, but also migration and proliferation of endothelial cells and VSMCs [15-18]. CXCL10 has been studied extensively in cardiovascular diseases, both experimentally and clinically.

The aim of this review is to summarize the role of CXCL10 in cardiovascular disease in both experimental and clinical studies and highlight the discrepancies between the different settings.

\section{CXCL10 Signaling and Effects}

2.1. CXCL10 Signaling. CXCL10 signals via binding to its receptor CXCR3. Next to CXCL10, CXCL9 and CXCL11 can also bind to this receptor. CXCR3 is a seven trans-membranespanning G-protein-coupled receptor (GPCR). This receptor is composed of the $\mathrm{G} \alpha, \mathrm{B} \beta$, and $\mathrm{G} \gamma$ subunit. Binding of a ligand to CXCR3 leads to the exchange of guanosine triphosphate (GTP) to guanosine phosphate (GDP), which is followed by dissociation of the regulatory $\mathrm{G} \alpha$ subunit from the catalytic $\mathrm{G} \beta \gamma$ subunit dimer. Upon activation, the $\mathrm{G}$ protein subunits can activate different enzymes leading to the production of inositol phosphates, protein kinase activation, an increase in intracellular $\mathrm{Ca}^{2+}$ production, and actin reorganization. Activation of the CXCR3 by CXCL10 leads to different cellular actions, such as chemotaxis, phagocytosis, cell degranulation, and respiratory burst [10, 43, 44]. Signaling via CXCR3 after CXCL10 binding is dependent on the type of target cell and the type of CXCR3 isoform bound to the surface of this cell.

The biological effects of CXCR3 signaling after CXCL10 between mice and humans are critically different. This is the result of differences in isoform expression in mice and humans. After the identification of CXCR3 expression in mice [45], no other isoforms are identified. In humans, the known isoforms identified are CXCR3-A, CXCR3-B, and CXCR3-alt. CXCR3-A consists of 368 amino acids and is associated with a $\mathrm{G} \alpha \mathrm{i}$ or a $\mathrm{G} \alpha \mathrm{q}$ subunit. It is widely expressed by different cell types. This isoform is similar to the CXCR3 in mice, regarding cell expression and signaling effects [46]. CXCR3-B is a larger receptor of 415 amino acids with a larger $\mathrm{N}$-terminus, compared to CXCR3-A. This isoform is mainly expressed by (microvascular) endothelial cells [44]. The third isoform,
CXCR3-alt, is generated by posttranscriptional exon skipping resulting in only four to five trans-membrane domains and shows a drastically altered C-terminal protein sequence. Its functions are relatively unknown, except that it is coexpressed with CXCR3-A at very low levels and that CXCL10 does not bind to this isoform and only mediates functions of CXCL11 [47].

As mentioned, CXCL10 can also exert its functions via CXCR3-independent pathways, mostly studied in an in vitro setting. CXCL10 is able to bind to GAGs [6] and is involved in inhibiting endothelial cell proliferation, independent of CXCR3 signaling [13]. The angiostatic properties of CXCL10, however, seem to be dependent on CXCR3 binding and not binding by GAGs [48]. Fibroblast recruitment by CXCL10 has also been linked to binding to GAGs instead of CXCR3, in which CXCL10 functions as an antifibrotic chemokine [12]. Interestingly, evidence has been found for CXCL10 signaling independent of binding to CXCR3 or GAGs, which might be related to epithelial and endothelial cell functions. The exact mechanism is not described yet [14].

2.2. CXCL10 Effects. CXCL10 can function via autocrine or paracrine effects. CXCL10 has versatile biological functions on different cell types, which are mostly dependent on the expression of CXCR3. Actions include attraction of inflammatory cells, such as monocytes and T lymphocytes, but CXCL10 can also function in proliferation and migration of endothelial cells and VSMCs [15-18, 49].

2.2.1. CXCL10 Effects in Mice. In mice, one isoform of CXCR3 receptor is identified as mediating angiostatic effects of CXCL10, similar to the CXCR3-A receptor identified in humans [46]. Effects of CXCL10 in mice have been described extensively. These are Th1 lymphocyte recruitment, activation and attraction of B lymphocytes, macrophages, and natural killer (NK) cells to the site of inflammation [43]. Effects of CXCL10 on endothelial cells and VSMCs have rarely been described in mice. In rats, however, Wang et al. described upregulation of CXCL10 by VSMCs and reported both proliferative and chemotactic effects of CXCL10 on VSMCs [15].

2.2.2. CXCL10 Effects in Humans. In humans, the effects of CXCL10 differ from those in mice. This is largely due to discrepancies in CXCR3 isoform expression between human and mice. The variety of effects of CXCL10 primarily depends on binding to CXCR3 and is therefore cell dependent. In humans, three isoforms of this receptor have been identified to which CXCL10 binds: CXCR3-A and CXCR3-B, differentially expressed by various cell types.

The first discovered isoform, CXCR3-A, is formed after the splicing of a single intron. CXCR3-A is known as the "angiostatic" isoform and is expressed by leukocytes $[46,50]$ and (vascular) SMCs [51, 52] and epithelial cells [53]. Functions of CXCR3-A are comparable to the CXCR3 functions in mice. Expression of the CXCR3-B or CXCR3alt isoform has not been described in mice, suggesting that the "antiangiogenic" properties of CXCR3 only emerge in humans [18]. After binding to CXCR3-A, CXCL10 can 


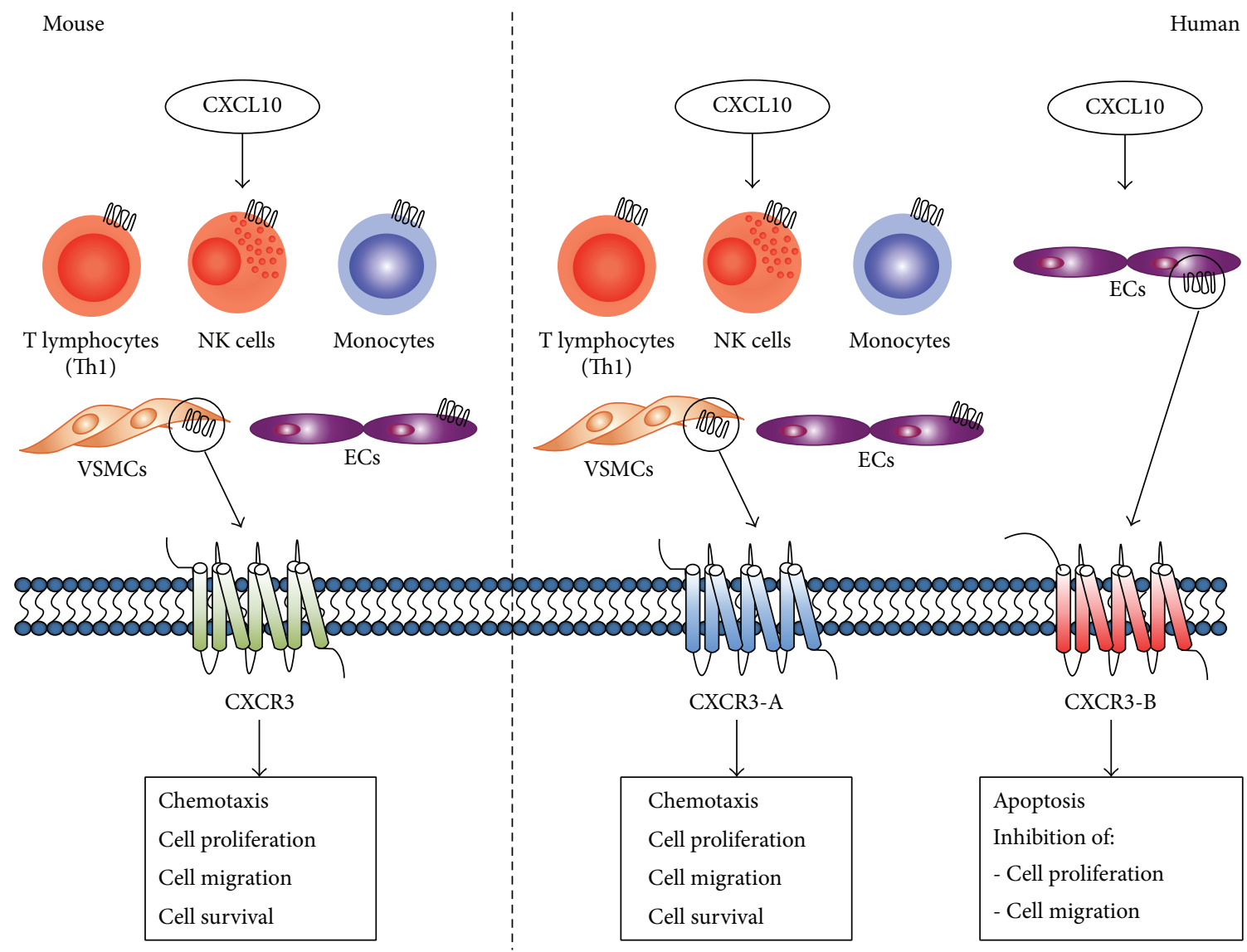

FIgURE 1: The effect of CXCL10 on CXCR3 isoforms in mouse and human tissues. Schematic overview of CXCR3 isoform expression in mouse and human cells and its actions after CXCL10 binding. In mice, one isoform of CXCR3 receptor has been identified and known as an angiostatic receptor. CXCR3 is expressed by T lymphocytes (Th1), monocytes, NK cells, VSMCs and endothelial cells (low expression levels). After binding of CXCL10, the murine CXCR3 receptor mediates cell functions, such as chemotaxis, cell proliferation, migration, and survival. In humans, this isoform is known as CXCR3-A with similar expression patterns and functions. In addition, a second isoform, known as CXCR3-B, is identified in human binding CXCL10. This isoform is primarily expressed by endothelial cells and is known for its antiangiogenic properties. These include promoting cell apoptosis and inhibiting cell proliferation and migration. CXCL10: chemokine (c-X-c motif) ligand 10; CXCR3: chemokine (c-X-c motif) receptor 3; ECs: endothelial cells; NK cell: natural killer cell; VSMCs: vascular smooth muscle cells.

promote cell proliferation and functions as a chemoattractant for leukocytes, especially Th1 lymphocytes [43]. CXCL10 has also recently been described in VSMC dedifferentiation during spiral artery remodeling in human VSMC cell line [54]. CXCR3-B, the "antiangiogenic" isoform, is primarily expressed by epithelial and endothelial cells. Binding of CXCL10 to CXCR3-B inhibits cell migration and promotes cell apoptosis. These data emerge from in vitro experiments performed with different human vascular endothelial cells, such as human umbilical cord endothelial cells (HUVECs) or human microvascular endothelial cells (HMECs) [46, 55-57].

The schematic Figure 1 summarizes the effects of CXCL10 on different cell types after binding to the different CXCR3 isoforms in both mouse and human.

\section{CXCL10 in Cardiovascular Disease}

3.1. Chemokines in Cardiovascular Disease. Vascular remodeling can be a consequence of (cardio)vascular disease and describes structural adaptation (enlargement or contraction) of the vascular lumen and vascular wall in response to various stimuli. These can be changes in blood flow leading to shear stress and hypoxia or immunological or mechanical changes leading to vascular wall damage $[1,58]$. During vascular remodeling, structural changes occur in all layers of the vascular wall. These changes can result from disturbance of the structural and functional integrity of the endothelium, accumulation of inflammatory cells, and changes in SMC composition, all contributing to the severity of the disease $[50,51]$.

Cellular behavior in vascular tissues is directed by chemokines. Different types of chemokines and their receptors have been described to be involved in vascular remodeling. CXCL10 is known to contribute to the pathophysiology of cardiovascular disease, such as atherosclerosis, aneurysm formation, $\mathrm{MI}$, and $\mathrm{PAD}$, in both experimental and clinical studies.

3.2. CXCL10 in Atherosclerosis. Atherosclerosis is a progressive inflammatory disease occurring in the middle-sized and 
larger arteries that can result in gradual luminal narrowing or acute thrombotic occlusion as a result of atherosclerotic plaque rupture [59]. Initially, activated endothelial cells express adhesion molecules resulting in adhesion and infiltration of inflammatory cells, such as monocytes and $\mathrm{T}$ lymphocytes. During plaque progression, inflammatory cells infiltrate the vessel wall and VSMCs start to proliferate and migrate. Chemokines in general have been described to be crucial during all phases of atherosclerotic disease progression $[60,61]$. The role of CXCL10 in atherosclerosis has been studied in the past. Mach et al. were of the first to describe expression of CXCL10 in human atherosclerotic plaques in different stages of the disease. Endothelial cells, VSMCs, and macrophages express CXCL10 at all examined stages of lesion development. Expression of CXCR3 could also be observed in these stages, and the vast majority of CXCR3 expressing cells was CD $4^{+} \mathrm{T}$ lymphocytes [62]. Shortly after this observation, mouse models for atherosclerosis revealed a role for CXCL10 in (early) lesion development. Compared to ApoE ${ }^{-/-}$mice, double knockout mice for ApoE and CXCL10 demonstrated significant reductions in atherogenesis, resulting in smaller lesions. Furthermore, less CD4+ $\mathrm{T}$ lymphocytes accumulated in the lesions, with a simultaneous increase of regulatory $\mathrm{T}$ lymphocytes $\left(\mathrm{T}_{\text {regs }}\right)$ [18]. $\mathrm{ApoE}^{-/-} \mathrm{CXCR}^{-/-}$mice also displayed significantly reduced atherosclerotic plaque development compared to $\mathrm{ApoE}^{-/-}$ mice, accompanied with increased numbers of $\mathrm{T}_{\text {regs }}[21]$. As a result, interventions targeting plaque progression have been tested to elucidate the effect of CXCL10 inhibition in vivo. Treatment with a specific antagonist for CXCR3 (NBI-74330) in $\mathrm{ApoE}^{-/-}$mice resulted in similar effects as in $\mathrm{CXCR}^{-/-}$ mice [22]. Also, treatment of $\mathrm{LDLR}^{-/-}$mice with an inhibitor for CCR5 and CXCR3 reduced atherosclerotic plaque area, $\mathrm{T}$ lymphocyte number, and IFN $\gamma$ plaque expression [23]. $\mathrm{ApoE}^{-/-}$mice subjected to shear stress followed by treatment with an antibody against CXCL10 resulted in a more stable plaque phenotype with twice as many SMCs compared to untreated controls but did not change overall plaque size [19]. Cheng et al. already observed the relation between shear stress, CXCL10 production, and plaque stability. Low shear stress resulted in a 10-fold higher CXCL10 mRNA expression in the vessel wall. Expression of CXCL10 was confirmed by immunohistochemical analysis, revealing that CXCL10 was mainly localized in the medial regions of the murine plaques [20]. Lastly, CXCL10 is significantly involved in VSMC proliferation and intimal hyperplasia, both important in arterial restenosis $[63,64]$.

3.3. CXCL10 in Aneurysm Formation. Aneurysm formation often coexists with advanced atherosclerotic disease [65] and systemic atherosclerosis is considered a risk factor for aneurysm formation [66]. Similar to atherosclerosis, aneurysmal tissue is characterized by inflammatory cell infiltrates, in particular B and T lymphocytes (mostly Th1) [67], which is related to aneurysm development and local thrombus formation [68]. Both inflammation and intraluminal thrombus formation have been related to aortic wall disruption and can therefore contribute to abdominal aortic aneurysm (AAA) growth and rupture [69]. Inflammatory cytokines and chemokines have been considered to play a causal role in aneurysm formation. CXCL10 and its receptor CXCR3 have been studied in mouse models for AAA, with conflicting results. King et al. observed that $\mathrm{ApoE}^{-/-} \mathrm{CXCL10}^{-/-}$mice after angiotensin II infusion had worse aneurysmal disease accompanied with more dilation and rupture, suggesting a protective role for CXCL10 in AAA formation [24]. In contrast, CXCR3 signaling itself seems to be crucial for aneurysm development in wildtype mice [26]. However, these results emerge from aneurysm formation in a different vessel type (aorta versus carotid artery). Contradictory, in a different murine model for aortic aneurysm formation, CXCR3 does not seem to play a crucial role [25]. In clinical studies, CXCL10 and CXCR3 expression have been related to recruitment of CXCR3+ T lymphocytes secreting IFN $\gamma$ and CXCL10. Furthermore, a correlation could be observed with outward arterial remodeling and intimal expansion. This remodeling resulted in matrix degradation [33]. Furthermore, differences were observed in inflammation between types of aneurysms, since CXCL10 is expressed 20-fold higher in AAA compared to popliteal artery aneurysms (PAA) [34]. Lastly, patients suffering from thoracic aortic aneurysms showed significantly elevated circulating CXCL10 levels compared to controls [26]. Although preclinical data show conflicting results, clinical studies point out a more clear direction of CXCL10 in aneurysm formation.

3.4. CXCL10 in Myocardial Infarction. During myocardial infarction (MI), upregulation of chemokines is a prominent feature during the postinfarction period. Several CXC chemokines are consistently upregulated in different MI models, where they play a crucial role in leukocyte trafficking and postinfarct wound healing [27]. The exact mechanisms of chemokine expression regulation are still unclear. In addition, upregulation of different chemokines at different time points during and after MI has not been fully elucidated [70]. Experimental studies have shown that CXCL10 not only functions as an angiostatic protein, but also acts as antifibrotic $[5,6,71]$. In particular, the latter finding may be crucial in postinfarct tissue healing and the accompanied fibrosis. In canine and murine MI models, CXCL10 was upregulated in the ischemic myocardial tissue during the first 24 hours [27, 28]. CXCL10 can therefore act as an angiostatic and antifibrotic chemokine to prevent premature neovascularization and fibrosis until the damaged myocardium is cleared from apoptotic and necrotic cells. Studying cardiac injury, cardiac repair, and postinfarct remodeling in $\mathrm{CXCL10} 0^{-/-}$mice after MI suggested an essential role for CXCL10 in the infarct healing. CXCL10 ${ }^{-/-}$mice showed more intense inflammatory cell infiltration, cardiac remodeling, and expansion of the formed scar. The fibrotic response was also attenuated and premature compared to wildtype mice. Surprisingly, CXCL10 ${ }^{-/-}$mice did not show early neovascularization [29]. CXCL10 has previously been studied as a circulating biomarker and predictor of cardiovascular damage in patients suffering from (acute) MI. The 
use of CXCL10 as a biomarker in cardiovascular disease will be discussed further in this review.

3.5. CXCL10 in Collateral Artery Formation. Patients suffering from PAD often have a reduced arterial flow to the lower limbs due to local atherosclerotic plaques in the middlesized and larger arteries. Adaptive collateral artery growth, known as arteriogenesis, is often hampered in these patients $[72,73]$. The underlying mechanism of arteriogenesis is not yet fully understood, but important processes involved are local infiltration and extravasation of inflammatory cells and proliferation and migration of VSMCs [74, 75]. Chemokines are key players in arteriogenesis and have already been extensively studied (for review, see Shireman [76]). Experimental models studying arteriogenesis already proposed different chemokines involved, such as monocyte chemoattractant protein (MCP)-1, also known as CCL2 [30, 77, 78]. Earlier studies already showed the role of CXCR3 and IFN $\gamma$ in hindlimb ischemia models. $\mathrm{CXCR}^{-/-}$mice undergoing unilateral femoral artery ligation resulted in lower perfusion recovery, accompanied with lower capillary density in the ischemic calf muscle and less infiltration of macrophages and $\mathrm{T}$ lymphocytes in the perivascular space in the ischemic hindlimb muscles [31]. Lee et al. showed that CXCL10 is upregulated in the hindlimb muscle tissue in the late phase of hindlimb ischemia, again suggesting the involvement of CXCL10 in neovascularization [30]. Recently, we confirmed the involvement of CXCL10 in arteriogenesis in a murine hindlimb ischemia model. Compared to wildtype, $\mathrm{CXCL}_{10}{ }^{-/-}$mice showed significantly reduced perfusion recovery after unilateral femoral artery ligation. This was explained by significantly less collateral vessel formation and hampered enlargement of collateral vasculature. In addition, we showed that in particular CXCL10 is involved in migration of VSMCs in vitro [32].

3.6. CXCL10 as a Biomarker for Cardiovascular Disease. As described above, CXCL10 is involved in different levels of cardiovascular disease severity. In the past, clinical studies have shown that circulating chemokine levels can function as independent predictors ("biomarkers") of (acute) ischemic cardiovascular events and cardiovascular death [79-82]. It is important to mention that a biomarker is only reliable for its function if it fulfills certain criteria, such as stability between individuals, long half-life, and easy and fast measurement using low-cost methods, and is not dependent of collection methods (e.g., serum/plasma) [83].

The function of CXCL10 as a biomarker for cardiovascular events has been investigated by measuring the transient levels of circulating CXCL10 protein during (acute) MI and treatment with percutaneous coronary intervention (PCI). In patients suffering from acute MI (AMI) CXCL10 serum levels correlated negatively to creatine kinase (CK)-MB release, a marker for MI. CXCL10 serum levels before PCI were considered as an independent predictor of CK-MB release. Furthermore, CXCL10 serum levels also negatively correlated to infarct size. Compared to healthy controls, AMI patients had significantly higher CXCL10 serum levels [40].
In contrast, in a study from Ørn et al. CXCL10 serum levels measured at the onset of an AMI correlated positively to infarct size, which did not confirm earlier data [41]. However, these studies used different methods to determine myocardial damage (CK-MB release versus Troponin I release). On the other hand, Herder et al. investigated CXCL10 serum levels along with other chemokines in a case-cohort study with patients diagnosed with coronary heart disease (CHD). The cohort consisted of 381 cases versus 1977 controls included over an 11-year time period. Although baseline CXCL10 serum levels were significantly higher in cases versus controls, adjustment for sex, age, and cardiovascular risk factors resulted in a nonsignificant contribution of CXCL10 serum measurements for risk assessment of CHD [35]. Furthermore, Ardigo et al. [37] identified CXCL10 as a potential biomarker in a much smaller cohort using a multibiomarker approach in patients suffering from CAD participating in the ADVANCE study. They report significantly higher serum CXCL10 levels in patients compared to controls (48 patients versus 44 controls). Nevertheless, measuring multiple chemokines is recommended for a more relevant biological analysis of the disease, instead of a single measurement approach of, for instance, CXCL10.

Simultaneously, Rothenbacher et al. reported a positive association between CXCL10 serum levels and risk for CHD in a clinical case-control study of almost 800 patients, even after adjustment for conventional CHD risk factors. Unfortunately, no (long-term) clinical followup was performed in these individuals [36]. Clinical studies provided important evidence for the role of CXCL10 in patients suffering from coronary artery disease (CAD). Patients suffering from unstable angina showed an increased CXCL10 expression in PBMCs as early as 6 hours after onset of complaints compared to controls or patients suffering from stable angina [38]. Furthermore, plasma CXCL10 and IFN $\gamma$ levels have been associated with the formation of collaterals in CAD patients [39].

Patients suffering from critical limb ischemia also showed higher serum CXCL10 levels, next to increased levels of other inflammatory markers. In addition, they showed a negative correlation between inflammatory cytokines and CD34+ bone marrow cells. Teraa et al. discussed that prolonged exposure to proinflammatory stimuli may lead to exhaustion or suppression of the CD34+ cell pool in the bone marrow [42]. It is important to mention that the measured circulating CXCL10 levels in different studies mostly showed a large variation, which is an important indicator of a low reliability of this approach. Because single CXCL10 measurements did not result in reliable predictions, the described studies all recommended a combined chemokine approach for cardiovascular risk prediction. However, this approach seems unrealistic from a practical and clinical point of view for obvious reasons, such as variability and costs. Although chemokines have been extensively described as pathogenic key players in cardiovascular disease, this does not automatically mean that they are suited as biomarkers or risk predictors. Furthermore, the use of chemokines as biomarkers in cardiovascular risk prediction brings some challenges. As mentioned, a biomarker needs to fulfill criteria 


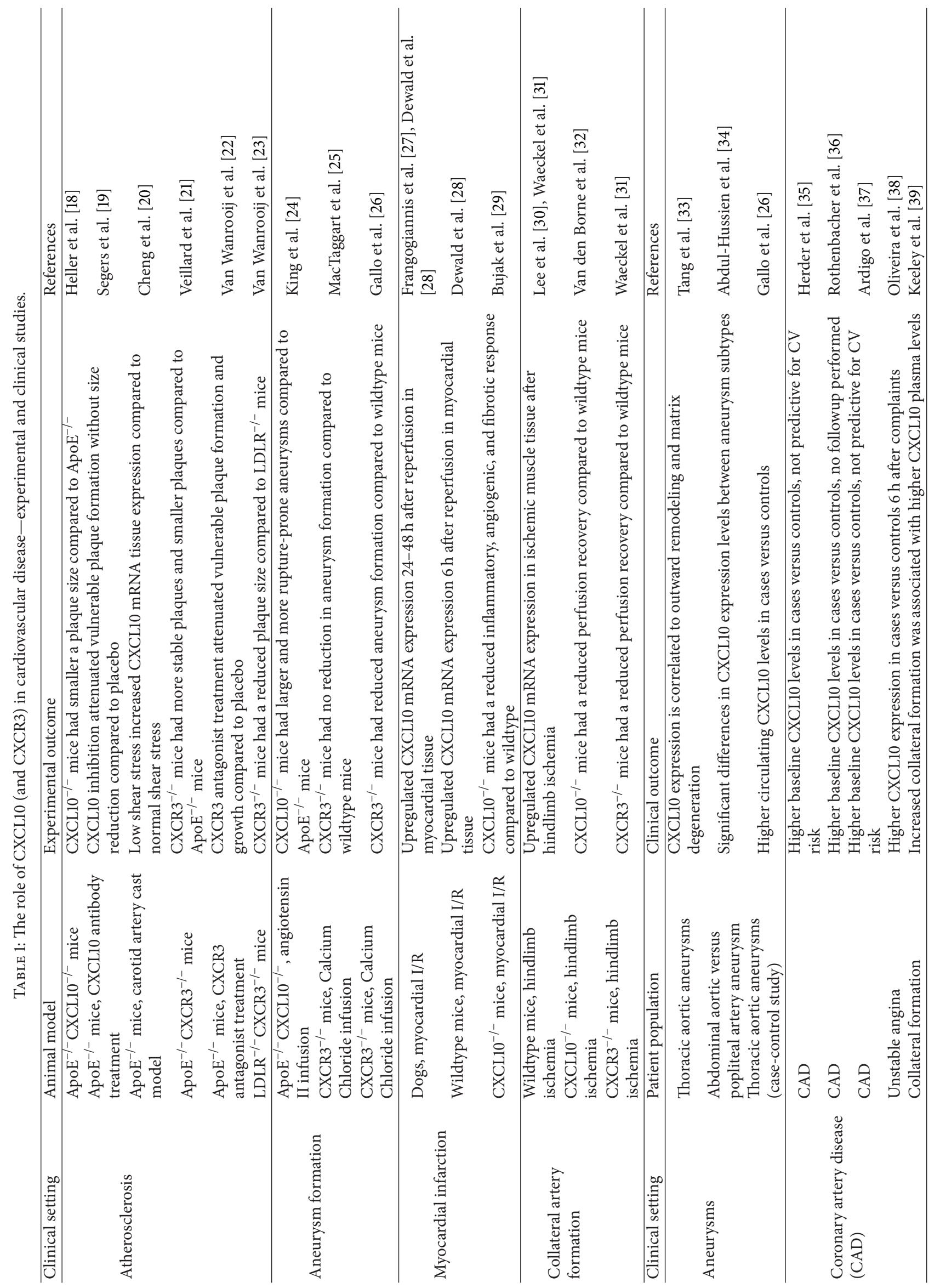




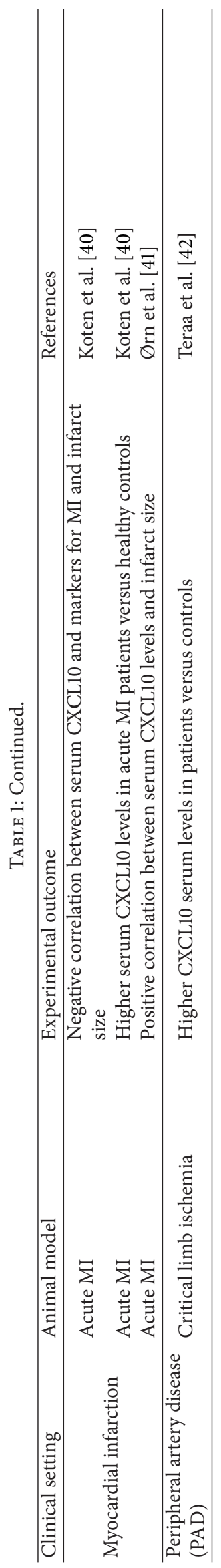


regarding its reliability. Chemokines as biomarkers are challenged by their stability, half-life, level fluctuation with use of different anticoagulants, and their low concentrations [83]. CXCL10 has been described to bind locally to endothelial cells or extracellular matrix components via GAGs, which makes the function of CXCL10 as a reliable circulating biomarker questionable.

In the past, genetic variations in chemokine genes have been extensively described, for example, for MCP-1, CCL2, and 5 (for reviews, see $[83,84]$ ). The mechanism by which the 9p21 locus is associated with CAD and other cardiovascular diseases has been investigated in the past by, for instance, the effect on impaired IFN $\gamma$ responses [85]. However, this mechanism does not involve the regulation of CXCL10 responses itself [86]. Until now, no genetic variations in the CXCL10 gene have been linked to cardiovascular risk prediction.

Table 1 provides an overview of the role of CXCL10 in cardiovascular disease in experimental and clinical settings.

\section{Summary and Conclusion}

In this review, we summarized the role of CXCL10 in different cardiovascular diseases, in both the experimental and clinical setting. The role of CXCL10 is rather complex, also depending on its action on different isoforms of the CXCR3 receptor. Since CXCR 3 is differentially expressed by different cell types, which also differs between mouse and man, translating experimental and clinical data is challenging. Also other signaling pathways, independent of CXCR3, have been described. The involvement of CXCL10 in atherosclerosis in both experimental and clinical settings suggests that CXCL10 contributes positively to the initiation and progression of the disease. In contrast, the functions of CXCL10 in aneurysm formation and MI are less consistent. This can be partly explained by study design and possible (biological) discrepancies between animal models and patient groups studied. Animal models for PAD suggest that CXCL10 is positively involved in arteriogenesis. Both CXCR3 and CXCL10 deficiencies resulted in decreased perfusion recovery in a murine hindlimb ischemia model. To this date, clinical evidence for the role of CXCL10 in PAD patients is scarce.

CXCL10 as a biomarker for cardiovascular risk prediction has been investigated in different clinical studies. However, measuring a single marker to predict cardiovascular risk will not be conclusive, which holds true for most potential biomarkers. Furthermore, CXCL10 as a reliable biomarker, regarding its stability, consistency, and possible binding to GAGs, is suboptimal. Lastly, CXCL10 is significantly involved in VSMC proliferation and intimal hyperplasia, both important in arterial restenosis. In particular, since other inflammatory diseases are also linked to CXCL10, further research is needed to elucidate the effects of CXCL10.

\section{Conflict of Interests}

The authors declare that there is no conflict of interests regarding the publication of this paper.

\section{Acknowledgments}

This research forms part of the Project P1.03 PENT of the research program of the Biomedical Materials Institute, cofunded by the Dutch Ministry of Economic Affairs, Agriculture, and Innovation. The financial contribution of the Dutch Heart Foundation is gratefully acknowledged.

\section{References}

[1] A. Schober, "Chemokines in vascular dysfunction and remodeling," Arteriosclerosis, Thrombosis, and Vascular Biology, vol. 28, no. 11, pp. 1950-1959, 2008.

[2] J. F. Bazan, K. B. Bacon, G. Hardiman et al., "A new class of membrane-bound chemokine with a CX3C motif," Nature, vol. 385, no. 6617, pp. 640-642, 1997.

[3] P. Romagnani, L. Lasagni, F. Annunziato, M. Serio, and S. Romagnani, "CXC chemokines: the regulatory link between inflammation and angiogenesis," Trends in Immunology, vol. 25, no. 4, pp. 201-209, 2004.

[4] A. Zlotnik and O. Yoshie, "The chemokine superfamily revisited," Immunity, vol. 36, pp. 705-716, 2012.

[5] R. M. Strieter, P. J. Polverini, D. A. Arenberg et al., "Role of C$\mathrm{X}-\mathrm{C}$ chemokines as regulators of angiogenesis in lung cancer," Journal of Leukocyte Biology, vol. 57, no. 5, pp. 752-762, 1995.

[6] R. M. Strieter, P. J. Polverini, S. L. Kunkel et al., "The functional role of the ELR motif in CXC chemokine-mediated angiogenesis," Journal of Biological Chemistry, vol. 270, no. 45, pp. 27348 27357, 1995.

[7] A. D. Luster and J. V. Ravetch, "Biochemical characterization of a $\gamma$ interferon-inducible cytokine (IP-10)," Journal of Experimental Medicine, vol. 166, no. 4, pp. 1084-1097, 1987.

[8] Y. Ohmori and T. A. Hamilton, "Cell type and stimulus specific regulation of chemokine gene expression," Biochemical and Biophysical Research Communications, vol. 198, no. 2, pp. 590596, 1994.

[9] Y. Ohmori and T. A. Hamilton, "The interferon-stimulated response element and a $\kappa \mathrm{B}$ site mediate synergistic induction of murine IP-10 gene transcription by IFN- $\gamma$ and TNF- $\alpha$," Journal of Immunology, vol. 154, no. 10, pp. 5235-5244, 1995.

[10] M. Loetscher, B. Gerber, P. Loetscher et al., "Chemokine receptor specific for IP10 and Mig: structure, function, and expression in activate T-Lymphocytes," Journal of Experimental Medicine, vol. 184, no. 3, pp. 963-969, 1996.

[11] B. A. D. Luster, S. M. Greenberg, and P. Leder, "The IP10 chemokine binds to a specific cell surface heparan sulfate site shared with platelet factor 4 and inhibits endothelial cell proliferation," Journal of Experimental Medicine, vol. 182, no. 1, pp. 219-231, 1995.

[12] D. Jiang, J. Liang, G. S. Campanella et al., "Inhibition of pulmonary fibrosis in mice by CXCL10 requires glycosaminoglycan binding and syndecan-4," Journal of Clinical Investigation, vol. 120, no. 6, pp. 2049-2057, 2010.

[13] G. S. V. Campanella, R. A. Colvin, and A. D. Luster, "CXCL10 can inhibit endothelial cell proliferation independently of CXCR3," PloS ONE, vol. 5, no. 9, p. e12700, 2010.

[14] K. Soejima and B. J. Rollins, "A functional IFN- $\gamma$-inducible protein-10/CXCL10-specific receptor expressed by epithelial and endothelial cells that is neither CXCR3 nor glycosaminoglycan," Journal of Immunology, vol. 167, no. 11, pp. 6576-6582, 2001. 
[15] X. Wang, T.-L. Yue, E. H. Ohlstein, C.-P. Sung, and G. Z. Feuerstein, "Interferon-inducible protein-10 involves vascular smooth muscle cell migration, proliferation, and inflammatory response," Journal of Biological Chemistry, vol. 271, no. 39, pp. 24286-24293, 1996.

[16] D. D. Taub, D. L. Longo, and W. J. Murphy, "Human interferoninducible protein-10 induces mononuclear cell infiltration in mice and promotes the migration of human T lymphocytes into the peripheral tissues of human peripheral blood lymphocytesSCID mice," Blood, vol. 87, no. 4, pp. 1423-1431, 1996.

[17] J. H. Dufour, M. Dziejman, M. T. Liu, J. H. Leung, T. E. Lane, and A. D. Luster, "IFN- $\gamma$-inducible protein 10 (IP-10; CXCL10)deficient mice reveal a role for IP-10 in effector T cell generation and trafficking," Journal of Immunology, vol. 168, no. 7, pp. 31953204, 2002.

[18] E. A. Heller, E. Liu, A. M. Tager et al., "Chemokine CXCL10 promotes atherogenesis by modulating the local balance of effector and regulatory T cells," Circulation, vol. 113, no. 19, pp. 2301-2312, 2006.

[19] D. Segers, J. A. Lipton, P. J. M. Leenen et al., "Atherosclerotic plaque stability is affected by the chemokine CXCL10 in both mice and humans," International Journal of Inflammation, vol. 2011, Article ID 936109, 9 pages, 2011.

[20] C. Cheng, D. Tempel, R. van Haperen et al., "Shear stressinduced changes in atherosclerotic plaque composition are modulated by chemokines," Journal of Clinical Investigation, vol. 117, no. 3, pp. 616-626, 2007.

[21] N. R. Veillard, S. Steffens, G. Pelli et al., "Differential influence of chemokine receptors CCR2 and CXCR3 in development of atherosclerosis in vivo," Circulation, vol. 112, no. 6, pp. 870-878, 2005.

[22] E. J. A. van Wanrooij, S. C. A. de Jager, T. van Es et al., "CXCR3 antagonist NBI-74330 attenuates atherosclerotic plaque formation in LDL receptor-deficient mice," Arteriosclerosis, Thrombosis, and Vascular Biology, vol. 28, no. 2, pp. 251-257, 2008.

[23] E. J. A. van Wanrooij, H. Happé, A. D. Hauer et al., "HIV entry inhibitor TAK-779 attenuates atherogenesis in lowdensity lipoprotein receptor-deficient mice," Arteriosclerosis, Thrombosis, and Vascular Biology, vol. 25, no. 12, pp. 2642-2647, 2005.

[24] V. L. King, A. Y. Lin, F. Kristo et al., "Interferon- $\gamma$ and the interferon-inducible chemokine CXCL10 protect against aneurysm formation and rupture," Circulation, vol. 119, no. 3, pp. 426-435, 2009.

[25] J. N. MacTaggart, W. Xiong, R. Knispel, and B. T. Baxter, "Deletion of CCR2 but not CCR5 or CXCR3 inhibits aortic aneurysm formation," Surgery, vol. 142, no. 2, pp. 284-288, 2007.

[26] A. Gallo, A. Saad, R. Ali, A. Dardik, G. Tellides, and A. Geirsson, "Circulating interferon- $\gamma$-inducible Cys-X-Cys chemokine receptor 3 ligands are elevated in humans with aortic aneurysms and Cys-X-Cys chemokine receptor 3 is necessary for aneurysm formation in mice," Journal of Thoracic and Cardiovascular Surgery, vol. 143, no. 3, pp. 704-710, 2012.

[27] N. G. Frangogiannis, L. H. Mendoza, M. Lewallen, L. H. Michael, C. W. Smith, and M. L. Entman, "Induction and suppression of interferon-inducible protein 10 in reperfused myocardial infarcts may regulate angiogenesis," The FASEB Journal, vol. 15, no. 8, pp. 1428-1430, 2001.

[28] O. Dewald, G. Ren, G. D. Duerr et al., "Of mice and dogs: species-specific differences in the inflammatory response following myocardial infarction," American Journal of Pathology, vol. 164, no. 2, pp. 665-677, 2004.
[29] M. Bujak, M. Dobaczewski, C. Gonzalez-Quesada et al., "Induction of the CXC chemokine interferon- $\gamma$-inducible protein 10 regulates the reparative response following myocardial infarction," Circulation Research, vol. 105, no. 10, pp. 973-983, 2009.

[30] C. W. Lee, E. Stabile, T. Kinnaird et al., “Temporal patterns of gene expression after acute hindlimb ischemia in mice: insights into the genomic program for collateral vessel development," Journal of the American College of Cardiology, vol. 43, no. 3, pp. 474-482, 2004.

[31] L. Waeckel, Z. Mallat, S. Potteaux et al., "Impairment in postischemic neovascularization in mice lacking the CXC chemokine receptor 3," Circulation Research, vol. 96, no. 5, pp. 576-582, 2005.

[32] P. van den Borne, R. T. Haverslag, M. Brandt et al., "Absence of chemokine (C-X-C Motif) ligand 10 diminishes perfusion recovery after local arterial occlusion in mice," Arteriosclerosis, Thrombosis, and Vascular Biology, vol. 34, pp. 594-602, 2014.

[33] P. C. Y. Tang, A. O. Yakimov, M. A. Teesdale et al., “Transmural inflammation by interferon- $\gamma$-producing $\mathrm{T}$ cells correlates with outward vascular remodeling and intimal expansion of ascending thoracic aortic aneurysms," The FASEB Journal, vol. 19, no. 11, pp. 1528-1530, 2005.

[34] H. Abdul-Hussien, R. Hanemaaijer, R. Kleemann, B. F. J. Verhaaren, J. H. van Bockel, and J. H. N. Lindeman, "The pathophysiology of abdominal aortic aneurysm growth: corresponding and discordant inflammatory and proteolytic processes in abdominal aortic and popliteal artery aneurysms," Journal of Vascular Surgery, vol. 51, no. 6, pp. 1479-1487, 2010.

[35] C. Herder, J. Baumert, B. Thorand et al., "Chemokines and incident coronary heart disease: results from the MONICA/KORA Augsburg case-cohort study, 1984-2002," Arteriosclerosis, Thrombosis, and Vascular Biology, vol. 26, no. 9, pp. 2147-2152, 2006.

[36] D. Rothenbacher, S. Müller-Scholze, C. Herder, W. Koenig, and H. Kolb, "Differential expression of chemokines, risk of stable coronary heart disease, and correlation with established cardiovascular risk markers," Arteriosclerosis, Thrombosis, and Vascular Biology, vol. 26, no. 1, pp. 194-199, 2006.

[37] D. Ardigo, T. L. Assimes, S. P. Fortmann et al., "Circulating chemokines accurately identify individuals with clinically significant atherosclerotic heart disease," Physiological Genomics, vol. 31, no. 3, pp. 402-409, 2007.

[38] R. T. D. D. Oliveira, R. L. Mamoni, J. R. M. Souza et al., "Differential expression of cytokines, chemokines and chemokine receptors in patients with coronary artery disease," International Journal of Cardiology, vol. 136, no. 1, pp. 17-26, 2009.

[39] E. C. Keeley, J. R. Moorman, L. Liu et al., "Plasma chemokine levels are associated with the presence and extent of angiographic coronary collaterals in chronic ischemic heart disease," PLoS ONE, vol. 6, no. 6, Article ID e21174, 2011.

[40] K. Koten, S. Hirohata, T. Miyoshi et al., "Serum interferongamma-inducible protein 10 level was increased in myocardial infarction patients, and negatively correlated with infarct size," Clinical Biochemistry, vol. 41, no. 1-2, pp. 30-37, 2008.

[41] S. Ørn, U. M. Breland, T. E. Mollnes et al., "The chemokine network in relation to infarct size and left ventricular remodeling following acute myocardial infarction," American Journal of Cardiology, vol. 104, no. 9, pp. 1179-1183, 2009.

[42] M. Teraa, R. W. Sprengers, P. E. Westerweel et al., "Bone marrow alterations and lower endothelial progenitor cell numbers in critical limb ischemia patients," PLoS ONE, vol. 8, Article ID e55592, 2013. 
[43] P. Romagnani and C. Crescioli, "CXCL10: a candidate biomarker in transplantation," Clinica Chimica Acta, vol. 413, pp. 1364-1373, 2012.

[44] C. Billottet, C. Quemener, and A. Bikfalvi, "CXCR3, a doubleedged sword in tumor progression and angiogenesis," Biochimica et Biophysica Acta, vol. 1836, pp. 287-295, 2013.

[45] B. Lu, A. Humbles, D. Bota et al., "Structure and function of the murine chemokine receptor CXCR3," European Journal of Immunology, vol. 29, pp. 3804-3812, 1999.

[46] L. Lasagni, M. Francalanci, F. Annunziato et al., "An alternatively spliced variant of CXCR3 mediates the inhibition of endothelial cell growth induced by IP-10, Mig, and I-TAC, and acts as functional receptor for platelet factor 4," Journal of Experimental Medicine, vol. 197, no. 11, pp. 1537-1549, 2003.

[47] J. E. Ehlert, C. A. Addison, M. D. Burdick, S. L. Kunkel, and R. M. Strieter, "Identification and partial characterization of a variant of human CXCR3 generated by posttranscriptional exon skipping," Journal of Immunology, vol. 173, no. 10, pp. 62346240, 2004.

[48] J. Yang and A. Richmond, "The angiostatic activity of interferon-inducible protein-10/CXCL10 in human melanoma depends on binding to CXCR3 but not to glycosaminoglycan," Molecular Therapy, vol. 9, no. 6, pp. 846-855, 2004.

[49] D. D. Taub, A. R. Lloyd, K. Conlon et al., "Recombinant human interferon-inducible protein 10 is a chemoattractant for human monocytes and $\mathrm{T}$ lymphocytes and promotes $\mathrm{T}$ cell adhesion to endothelial cells," Journal of Experimental Medicine, vol. 177, no. 6, pp. 1809-1814, 1993.

[50] M. Loetscher, P. Loetscher, N. Brass, E. Meese, and B. Moser, "Lymphocyte-specific chemokine receptor CXCR3: regulation, chemokine binding and gene localization," European Journal of Immunology, vol. 28, pp. 3696-3705, 1998.

[51] C. E. Brightling, A. J. Ammit, D. Kaur et al., "The CXCL10/CXCR3 axis mediates human lung mast cell migration to asthmatic airway smooth muscle," American Journal of Respiratory and Critical Care Medicine, vol. 171, no. 10, pp. 1103-1108, 2005.

[52] R. Saunders, A. Sutcliffe, D. Kaur et al., "Airway smooth muscle chemokine receptor expression and function in asthma," Clinical and Experimental Allergy, vol. 39, no. 11, pp. 1684-1692, 2009.

[53] Q. Wu, R. Dhir, and A. Wells, "Altered CXCR3 isoform expression regulates prostate cancer cell migration and invasion," Molecular Cancer, vol. 11, article 3, pp. 1-17, 2012.

[54] A. E. Wallace, J. E. Cartwright, R. Begum et al., “Trophoblastinduced changes in $\mathrm{C}-\mathrm{x}-\mathrm{C}$ motif chemokine 10 expression contribute to vascular smooth muscle cell dedifferentiation during spiral artery remodeling," Arteriosclerosis, Thrombosis, and Vascular Biology, vol. 33, pp. e93-e101, 2013.

[55] R. J. Bodnar, C. C. Yates, and A. Wells, "IP-10 blocks vascular endothelial growth factor-induced endothelial cell motility and tube formation via inhibition of calpain," Circulation Research, vol. 98, no. 5, pp. 617-625, 2006.

[56] R. J. Bodnar, C. C. Yates, M. E. Rodgers, X. Du, and A. Wells, "IP10 induces dissociation of newly formed blood vessels," Journal of Cell Science, vol. 122, no. 12, pp. 2064-2077, 2009.

[57] C. C. Yates-Binder, M. Rodgers, J. Jaynes et al., "An IP-10 (CXCL10)-derived peptide inhibits angiogenesis," PLoS ONE, vol. 7, Article ID e40812, 2012.

[58] J. J. Wentzel, F. J. H. Gijsen, N. Stergiopulos, P. W. Serruys, C. J. Slager, and R. Krams, "Shear stress, vascular remodeling and neointimal formation," Journal of Biomechanics, vol. 36, no. 5, pp. 681-688, 2003.

[59] G. K. Hansson, "Inflammation, atherosclerosis, and coronary artery disease," The New England Journal of Medicine, vol. 353, no. 4, pp. 429-430, 2005.

[60] P. Libby, "Inflammation in atherosclerosis," Arteriosclerosis, Thrombosis, and Vascular Biology, vol. 32, pp. 2045-2051, 2012.

[61] L. G. Spagnoli, E. Bonanno, G. Sangiorgi, and A. Mauriello, "Role of inflammation in atherosclerosis," Journal of Nuclear Medicine, vol. 48, no. 11, pp. 1800-1815, 2007.

[62] F. Mach, A. Sauty, A. S. Iarossi et al., "Differential expression of three $\mathrm{t}$ lymphocyte-activating CXC chemokines by human atheroma-associated cells," Journal of Clinical Investigation, vol. 104, no. 8, pp. 1041-1050, 1999.

[63] A. Kawamura, S.-I. Miura, M. Fujino et al., "CXCR3 chemokine receptor-plasma IP10 interaction in patients with coronary artery disease," Circulation Journal, vol. 67, no. 10, pp. 851-854, 2003.

[64] H. Zuojun, H. Lingyu, H. Wei et al., "Interference of IP-10 expression inhibits vascular smooth muscle cell proliferation and intimal hyperplasia in carotid artery: a new insight in the prevention of restenosis," Cell Biochemistry and Biophysics, vol. 62, no. 1, pp. 125-135, 2012.

[65] S. H. Johnsen, S. H. Forsdahl, K. Singh, and B. K. Jacobsen, "Atherosclerosis in abdominal aortic aneurysms: a causal event or a process running in parallel? The tromsø study," Arteriosclerosis, Thrombosis, and Vascular Biology, vol. 30, no. 6, pp. 1263$1268,2010$.

[66] F. A. Lederle, G. R. Johnson, S. E. Wilson et al., "Prevalence and associations of abdominal aortic aneurysm detected through screening," Annals of Internal Medicine, vol. 126, no. 6, pp. 441449, 1997.

[67] W. H. Pearce and A. E. Koch, "Cellular components and features of immune response in abdominal aortic aneurysms," Annals of the New York Academy of Sciences, vol. 800, pp. 175-185, 1996.

[68] A. Sagan, W. Mrowiecki, T. P. Mikolajczyk et al., "Local inflammation is associated with aortic thrombus formation in abdominal aortic aneurysms. Relationship to clinical risk factors," Journal of Thrombosis and Haemostasis, vol. 108, pp. 812-823, 2012.

[69] D. Koole, H. J. A. Zandvoort, A. Schoneveld et al., "Intraluminal abdominal aortic aneurysm thrombus is associated with disruption of wall integrity," Journal of Vascular Surgery, vol. 57, pp. 77-83, 2013.

[70] N. G. Frangogiannis and M. L. Entman, "Chemokines in myocardial ischemia," Trends in Cardiovascular Medicine, vol. 15, no. 5, pp. 163-169, 2005.

[71] H. Shiraha, A. Glading, K. Gupta, and A. Wells, "IP-10 inhibits epidermal growth factor-induced motility by decreasing epidermal growth factor receptor-mediated calpain activity," Journal of Cell Biology, vol. 146, no. 1, pp. 243-253, 1999.

[72] W. Schaper, "Collateral circulation. Past and present," Basic Research in Cardiology, vol. 104, no. 1, pp. 5-21, 2009.

[73] P. Carmeliet, "Mechanisms of angiogenesis and arteriogenesis," Nature Medicine, vol. 6, no. 4, pp. 389-395, 2000.

[74] D. Scholz, W. Ito, I. Fleming et al., "Ultrastructure and molecular histology of rabbit hind-limb collateral artery growth (arteriogenesis)," Virchows Archiv, vol. 436, no. 3, pp. 257-270, 2000.

[75] W. Cai and W. Schaper, "Mechanisms of arteriogenesis," Acta Biochimica et Biophysica Sinica, vol. 40, no. 8, pp. 681-692, 2008. 
[76] P. K. Shireman, "The chemokine system in arteriogenesis and hind limb ischemia," Journal of Vascular Surgery, vol. 45, no. 6, pp. 48-56, 2007.

[77] N. van Royen, I. Hoefer, I. Buschmann et al., "Effects of local MCP-1 protein therapy on the development of the collateral circulation and atherosclerosis in Watanabe hyperlipidemic rabbits," Cardiovascular Research, vol. 57, no. 1, pp. 178-185, 2003.

[78] M. Voskuil, I. E. Hoefer, N. van Royen et al., "Abnormal monocyte recruitment and collateral artery formation in monocyte chemoattractant protein-1 deficient mice," Vascular Medicine, vol. 9, no. 4, pp. 287-292, 2004.

[79] A. O. Kraaijeveld, S. C. A. de Jager, W. J. de Jager et al., "CC chemokine ligand-5 (CCL5/RANTES) and CC chemokine ligand-18 (CCL18/PARC) are specific markers of refractory unstable angina pectoris and are transiently raised during severe ischemic symptoms," Circulation, vol. 116, no. 17, pp. 1931-1941, 2007.

[80] G. Davì, A. Tuttolomondo, F. Santilli et al., "CD40 ligand and MCP-1 as predictors of cardiovascular events in diabetic patients with stroke," Journal of Atherosclerosis and Thrombosis, vol. 16, no. 6, pp. 707-713, 2009.

[81] F. Canouï-Poitrine, G. Luc, Z. Mallat et al., "Systemic chemokine levels, coronary heart disease, and ischemic stroke events: the PRIME study," Neurology, vol. 77, no. 12, pp. 1165-1173, 2011.

[82] S. C. A. de Jager, B. W. C. Bongaerts, M. Weber et al., "Chemokines CCL3/MIP1 $\alpha$, CCL5/RANTES and CCL18/PARC are independent risk predictors of short-term mortality in patients with acute coronary syndromes," PLoS ONE, vol. 7, Article ID e45804, 2012.

[83] P. Aukrust, B. Halvorsen, A. Yndestad et al., "Chemokines and cardiovascular risk," Arteriosclerosis, Thrombosis, and Vascular Biology, vol. 28, no. 11, pp. 1909-1919, 2008.

[84] P. Aukrust, A. Yndestad, C. Smith, T. Ueland, L. Gullestad, and J. K. Damås, "Chemokines in cardiovascular risk prediction," Thrombosis and Haemostasis, vol. 97, no. 5, pp. 748-754, 2007.

[85] O. Harismendy, D. Notani, X. Song et al., "9p21 DNA variants associated with coronary artery disease impair interferon- $\gamma 3$ signalling response," Nature, vol. 470, no. 7333, pp. 264-268, 2011.

[86] C. Erridge, J. Gracey, P. S. Braund, and N. J. Samani, “The 9p21 locus does not affect risk of coronary artery disease through induction of type 1 interferons," Journal of the American College of Cardiology, vol. 62, pp. 1376-1381, 2013. 


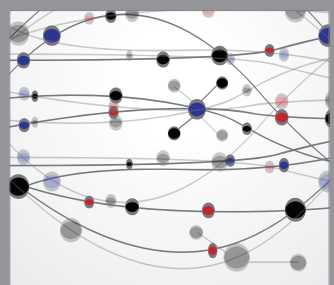

The Scientific World Journal
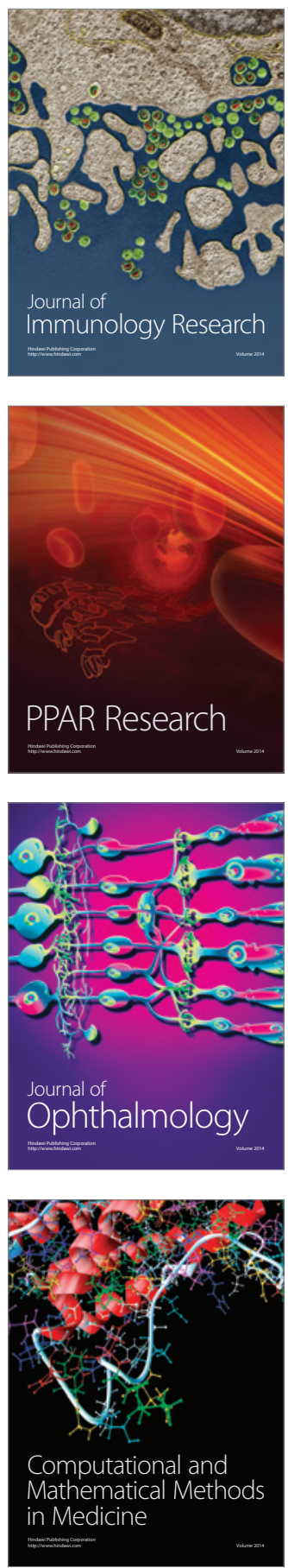

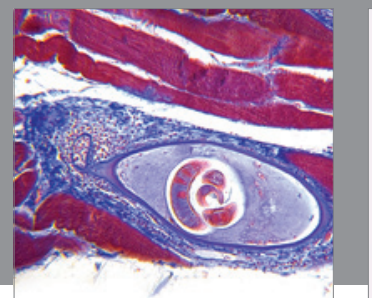

Gastroenterology

Research and Practice
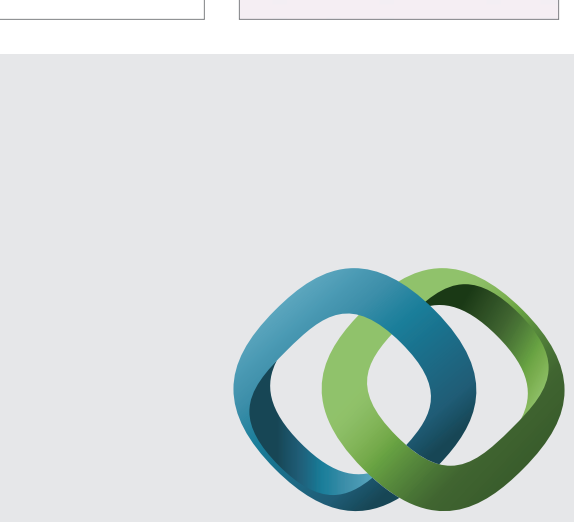

\section{Hindawi}

Submit your manuscripts at

http://www.hindawi.com
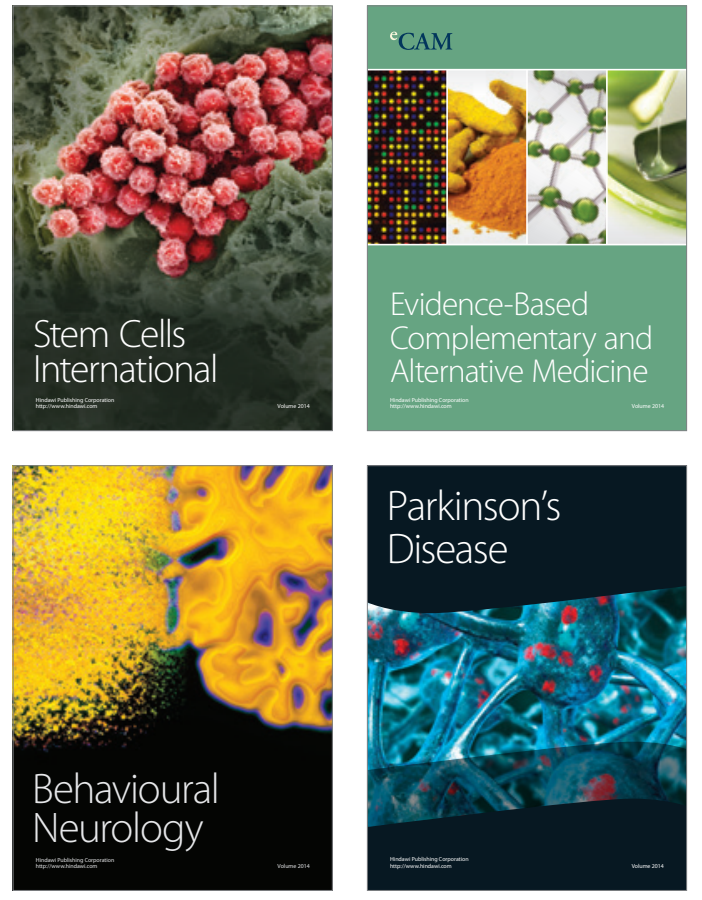
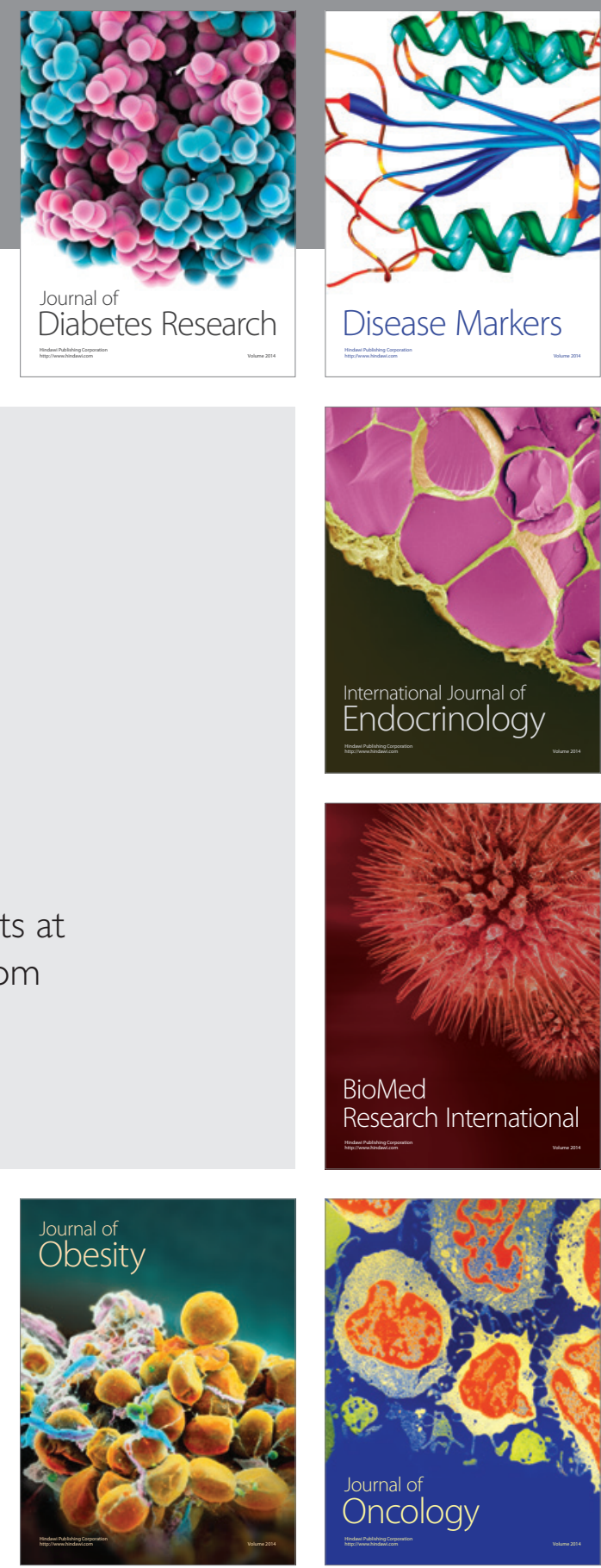

Disease Markers
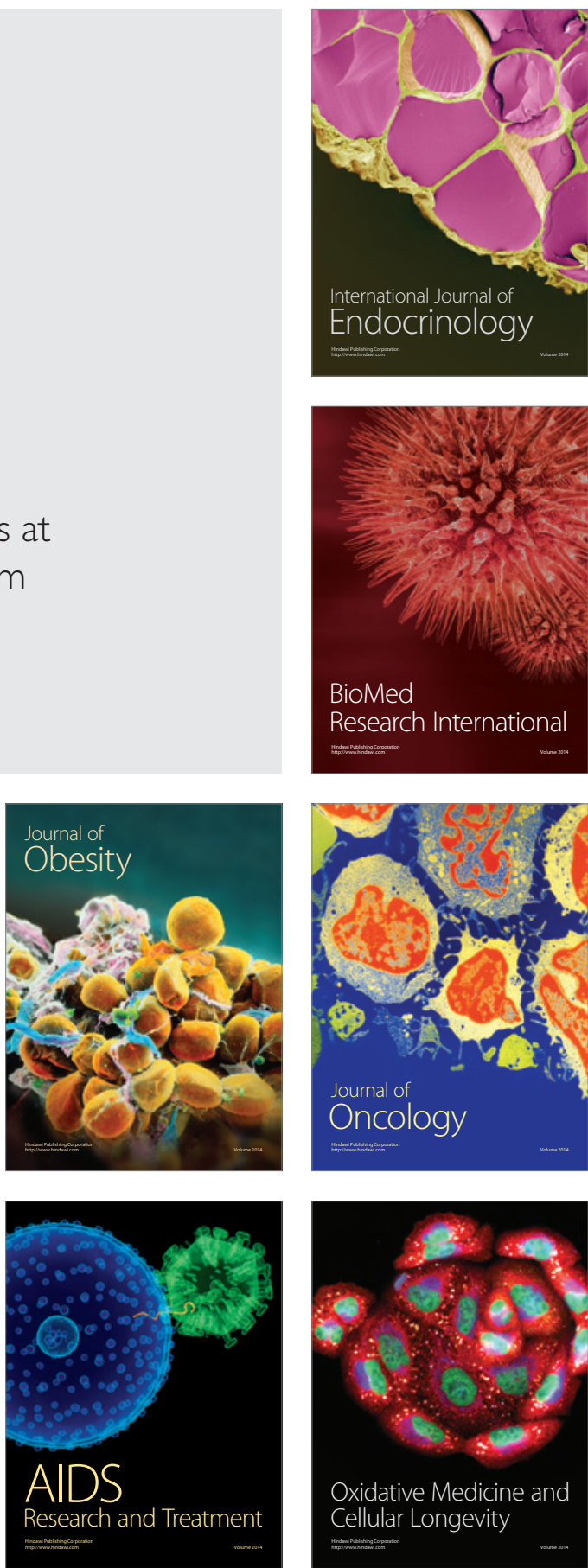